\title{
The Milky Way thin disk structure as revealed by stars and young open clusters
}

\section{Giovanni Carraro}

\author{
Alonso de Cordova 3107, 19001, Santiago de Chile, Chile \\ email: gcarraro@eso.org
}

\begin{abstract}
.
In this contribution I shall focus on the structure of the Galactic thin disk. The evolution of the thin disk and its chemical properties have been discussed in detail by T. Bensby's contribution in conjunction with the properties of the Galactic thick disk, and by L.Olivia in conjunction with the properties of the Galactic bulge. I will review and discuss the status of our understanding of three major topics, which have been the subject of intense research nowadays, after long years of silence: (1) the spiral structure of the Milky Way, (2) the size of the Galactic disk, and (3) the nature of the Local arm (Orion spur), where the Sun is immersed. The provisional conclusions of this discussion are that : (1) we still have quite a poor knowledge of the Milky Way spiral structure, and the main disagreements among various tracers are still to be settled; (2) the Galactic disk does clearly not have an obvious luminous cut-off at about $14 \mathrm{kpc}$ from the Galactic center, and next generation Galactic models need to be updated in this respect, and (3) the Local arm is most probably an inter-arm structure, similar to what we see in several external spirals, like M 74. Finally, the impact of Gaia and LAMOST in this field will be briefly discussed as well.
\end{abstract}

Keywords. Open clusters and associations: general - Galaxy: structure -Galaxy: evolution: Galaxy: disk

\section{Introduction}

A quick glance at the Hubble Atlas of Galaxies immediately reveals that spiral galaxies, when seen face-off, possess dusty and gaseous disks where stars are barely visible. On the other hand, when seen face-on, they exhibit quite spectacular structures in the form of gaseous and stellar spiral arms, bridges, inter-arm structures, knots, bifurcations, and so forth. These detailed shapes are hardly repeated from one spiral galaxy to the other.

Our Milky Way is believed to be a grand design spiral galaxy, of Hubble type $\mathrm{Sb}$ or Sc, and most probably very similar to NGC 1232, although its precise structure has been challenging us for more than 60 years, and it is still very far from being understood. The very special position of the Sun, close to the Galactic plane, and immersed in a spiral feature (the Orion Spur), is one of the major difficulties astronomers have to face, together with the fact that to probe the disk structure we have to search for features along the plane and penetrate across thick layers of gas and dust.

The thin disk of the Milky Way contains in fact mostly gas, dust, and stars. It is the place where star formation occurs, inside the high-density spiral arms. It is most probably detached from the Galactic thick disk and bulge (Gilmore et al. 1989), although there are different opinions on the subject (see the chapters by Bensby, Origlia, Lepine, and Rix in this volume).

Young stars (of spectral type $\mathrm{O}$ to A), either in clusters or in the general field, and gas (either atomic or molecular), are routinely used as tracers of its structure. In Table 1, I summarize the approximate distribution in mass of the typical thin disk components from Kalberla \& Kerp (2009), and references therein. In the mass budget, stars are the largest contributors. At odds with the Galactic thick disk, stars in the thin disk have a large spread in age, from virtually 
0 to about 10 Gyrs (see Bensby review). Stars as old as 10 Gyrs are no longer tracers of the Galactic thin disk structure as we observe it now, since they departed significantly from their birth-place due to a variety of dynamical processes (migration, spiral arm perturbations, disk crossing, encounters with molecular clouds, and so forth). The present-day structure of the thin disk is therefore better described by young stars and gas. These two tracers are confined in a thin layer, less than 100 parsec thick. Young stars, of OB spectral type, are typically clumped, since they mostly form in associations and star clusters. The latter have a typical life-time of a few hundred million years, and afterwards they dissolve into the general Galactic field (de la Fuente Marcos et al. 2013). Older clusters are found, especially in the outer disk (Carraro et al. 2013, and references therein), or at larger distances from the plane, where the environment is more favorable, and fewer encounters with molecular clouds are probable.

In this review I will focus on young stars and stellar clusters, and how they trace the actual structure of the Milky Way. The perspective is purely observational. I will refer from time to time also to $\mathrm{HI}$ and $\mathrm{CO}$ surveys without, however, entering into much detail. I am not going to mention much about maser surveys either (Reid et al. 2009). This is quite a promising, but still young, technique which surely will significantly impact our understanding of the disk in the future, once more data have been accumulated.

\section{The spiral structure of the Milky Way}

The quest for the spiral structure of the Milky Way started enthusiastically in the early fifties, almost 70 years ago. Interestingly enough, for the perspective of this talk, the quest started using spectrophotometric distances to OB stars, since astronomers at the time realized that these stars are present in the spiral arms of external galaxies (Morgan et al. 1952). The original picture of the Milky Way spiral structure (see Fig. 1) only contains the local (Orion) arm (spur), where the Sun is located, the Perseus arm in the second Galactic quadrant, and the Great Carina star forming region in the fourth Galactic quadrant.

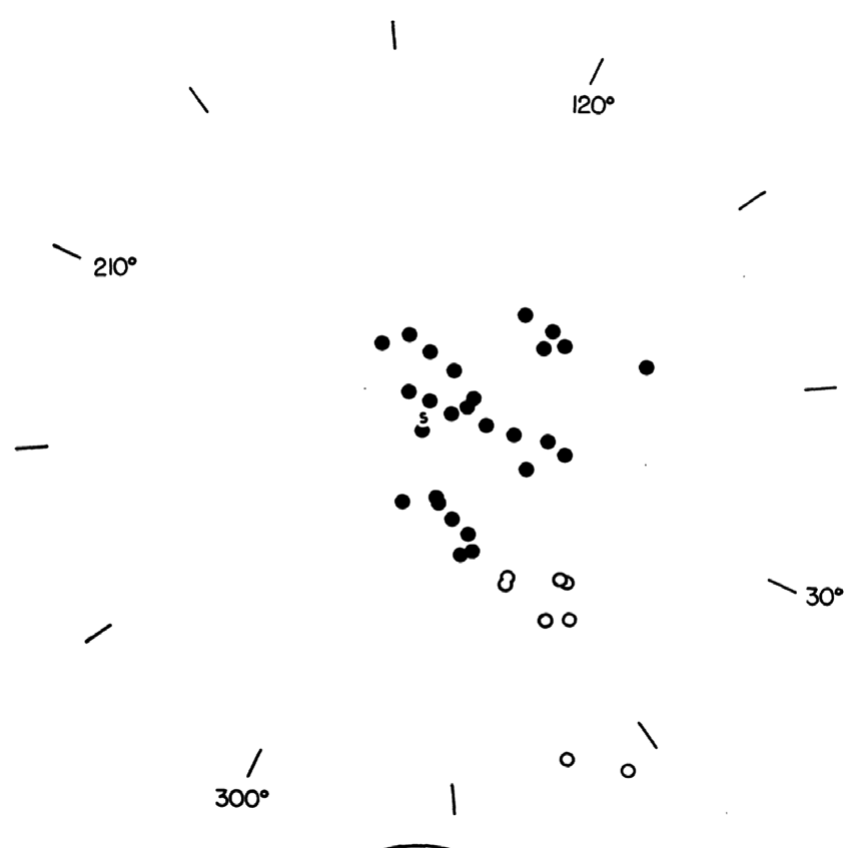

Figure 1. The original view of the Milky Way as traced by OB stars in the solar vicinity. Numbers indicate the Galactic longitude. Notice three clear structures: the Orion spur, where the Sun (S) is, the Carina Sagittarius arm toward the Galactic center, and the Perseus arm in the second quadrant, toward the anti-center. From Morgan et al. (1953). 


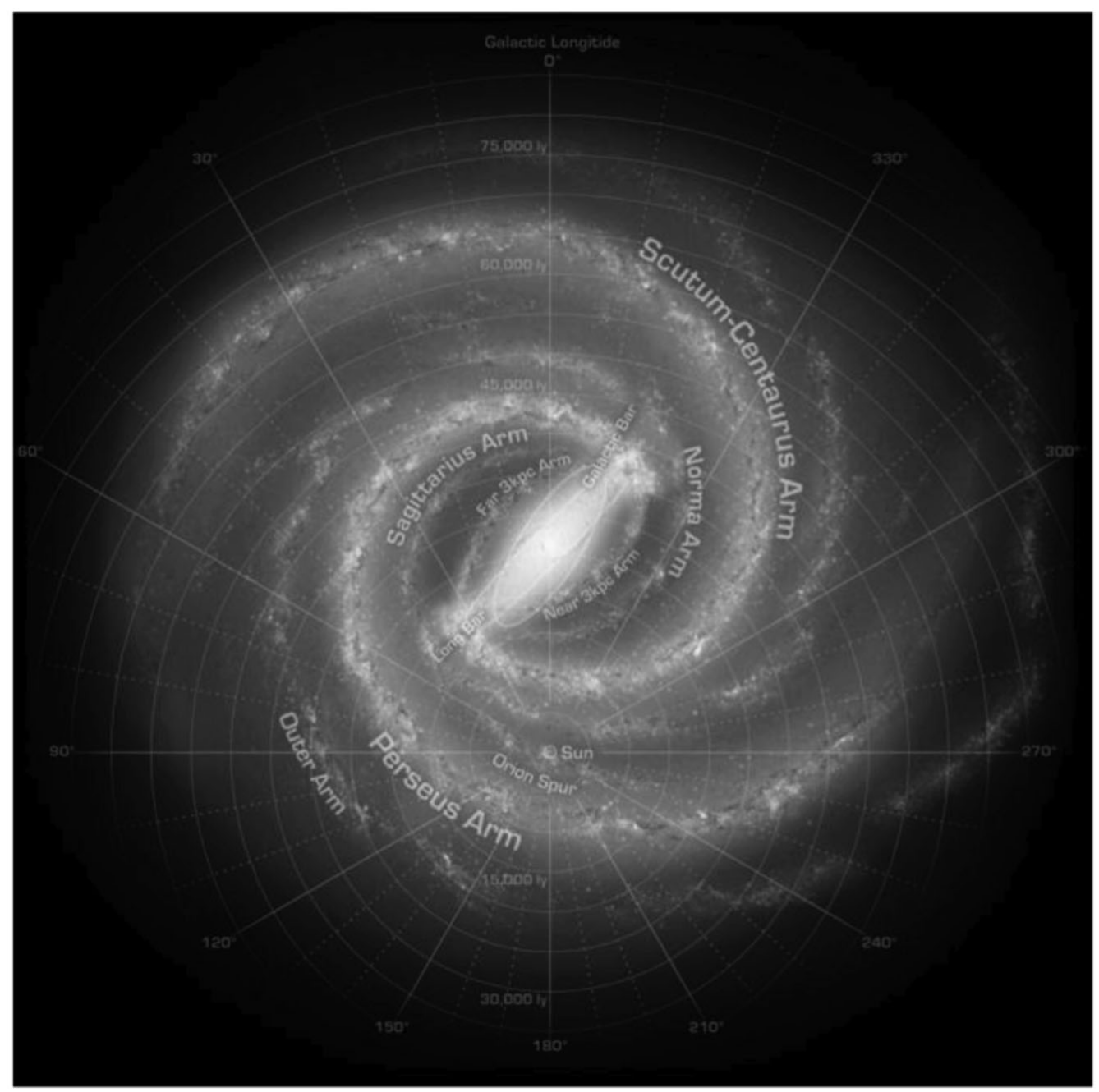

Figure 2. The GLIMPSE artistic-rendered view of the Milky Way spiral structure (Churchwell et al. 2009).

The HI $21 \mathrm{~cm}$ line was discovered later (for details, see the beautiful review from Gingerich 1985), but its success was so huge that it replaced the optical tracers almost completely in the quest. The HI view of the Milky Way spiral structure, developed after 50 years of research, is the one of a Galaxy with two major arms, the Perseus and the Scutum-Centaurus arms. This is summarized by the artistic rendering in Fig. 2, which results from star counts using Glimpse data (Churchwell et al 2009), filtered by HI data. The two major arms are clearly indicated, while arms like Carina or the outer (Norma Cygnus) arm appear as minor, secondary structures. Overall, the Galaxy appears as a beautiful, ordered, grand design spiral galaxy, of a kind that we very rarely see, e.g., by inspecting the Hubble Atlas. Another recent, but strikingly different, HI realization of the spiral structure of the Milky Way is in Levine et al. (2006).

Traditionally, this picture is in net contrast with the optical/HII view of the Galaxy, that postulates that the Milky Way is a four arm spiral galaxy. This constitutes the Georgelin \& Georgelin legacy, summarized in Russeil (2003), recently refined in much more detail -but just in the first Galactic quadrant - by Anderson et al. (2011).

According to Churchwell et al. (2009), the spectacular view of the Milky Way in Fig. 2 comes from counting red clump stars. These are typically 200-300 Myr or even older stars and, 
Table 1. Approximate distribution in mass of various thin disk components, taken from Kalberla \&Kerp (2009), and references therein.

\begin{tabular}{lc}
\hline Component & Mass $\left(10^{9} M_{\odot}\right)$ \\
\hline HI & 8.0 \\
HII & 2.0 \\
CO,CS & 2.5 \\
stars & 70.5 \\
\hline
\end{tabular}

technically speaking, are not the ideal tracers of the young, gas-rich, star forming regions which usually define spiral features. One can speculate that whatever stellar tracers can be in fact used, since stars tend to be trapped anyway in the high density potential wells generated by spiral perturbations (see also the discussion at the end of this document). Our opinion is that this is a weak argument. To illustrate it, we refer to the results from Dias \& Lepine (2005) in Figs. 3 and 4 . Open clusters in the solar volume for different ages are considered. From the left to the right one can readily appreciate how very young open clusters position in a seemingly regular spiral structure, while at increasing cluster ages, the spatial distributions get more scattered so that no structure can be detected, since these clusters had a lot of time to move away from their birth-places (see Fig. 3).

In recent years, the quest for spiral structure of the Milky Way using star and star clusters experienced quite a significant burst of activity. This is mostly because of the failure of HI studies, that cannot go much beyond the detection of gas density peaks in the velocity space (McClure-Griffiths et al. 2004; Dame et al 2011), and struggle in the process of translating these velocities into distances (Liszt 1985).

Young open clusters and OB stars are powerful spiral arm tracers, and can probe spiral features in very remote regions of the Milky Way. Of course, deriving their distance is not an easy task (Carraro 2011), but the technique is well established and suffers from less systematics than, for instance, red clump stars (see below). Deriving the distance to a young (less than 100 Myr) open cluster/ association is straightforward in a sense. Star clusters and OB associations are groups of coeval, co-spatial stars, and their distances can be robustly estimated in a statistical way. When $U B V$ photometry is combined with spectral classification, the extinction law toward a star cluster can be derived and, in turn, its distance measured (see, as an illustration, Carraro et al. 2013, where the distance to the young open cluster Westerlund 2 is derived). The systematic use of star clusters to map the spiral structure of the Milky Way has been limited so far only
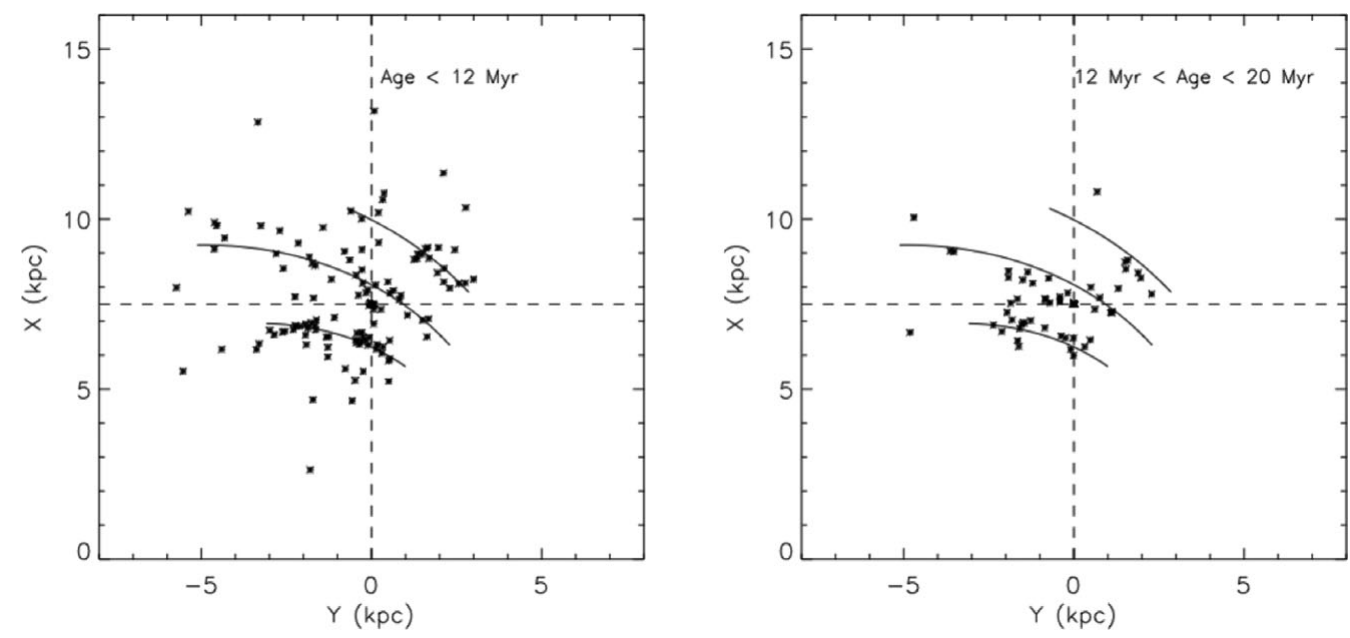

Figure 3. Spatial distribution of open clusters in the plane of the Milky Way as a function of their age. From Dias \& Lepine (2005). 


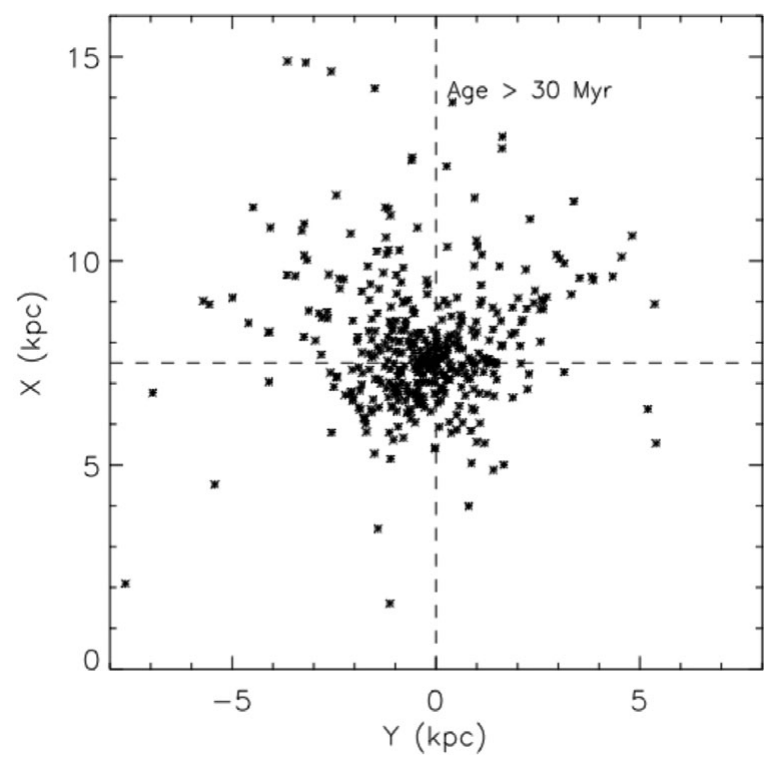

Figure 4. Spatial distribution of open clusters in the plane of the Milky Way as a function of their age. From Dias \& Lepine (2005).

to the third Galactic quadrant $\left(180^{\circ} \leqslant l \leqslant 270^{\circ}\right)$. In this sector of the Milky Way extinction is small (Moitinho 2001), and star clusters can be detected to very distant regions (Carraro et al. 2010), because the young disk is significantly warped (Moitinho et al 2006).

A major break-through is reported in Vázquez et al. (2008), where for the very first time, distances to a large sample of young (less than $100 \mathrm{Myr}$ ) open clusters (mostly from Moitinho 2001) are compared with CO clumps all the way to $20 \mathrm{kpc}$ from the Galactic center in the anticenter direction. Coupling homogenous photometry with a solid technique, this study unraveled the spiral structure in the third quadrant (see Fig. 5), showing that the Perseus arm does not seem to continue in this quadrant. Its structure is broken by the local arm (see below), which extends in the third quadrant all the way to the outer, Norma Cygnus arm. This is in contrast with the GLIMPE realization of the third quadrant, but in nice agreement, e.g., with the HI picture of the Milky Way from Levine et al. (2006). In fact, this study finds that the strongest arms in Hydrogen are not Perseus or Scutum, but possibly Norma-Cygnus and Carina-Sagittarius, and confirms the nature of the local arm.

Attempts to repeat this kind of analysis are ongoing in the second quadrant (Monguió et al 2012), using Stromgren ubvy photometry. This photometric system has the obvious advantage that stellar parameters can be measured precisely, but it is distance-limited due to the difficult to go deep enough with narrow band photometry. Besides, the dusty Perseus arm in the second Galactic quadrant prevents reaching much further away, at least in optical.

The general, well-known optical picture of the Milky Way spiral structure in the solar vicinity is confirmed also using Cepheid stars, for which distances can be measured with high precision (Majaess et al. 2009).

\section{Does the Galactic disk have a density cut-off?}

Models of Galactic structure and stellar population like the Besancon one (Robin et al. 2003) postulate that the Galactic disk has a strong density drop at about $14 \mathrm{kpc}$ from the Galactic center, in the anti-center direction. Recently, Minniti et al. (2011) found that this cut-off is a general feature, and it is detectable in several other Galactic directions (their Fig. 3). These results are clearly biased both in the underlying technique and in the assumptions. In the case of Minniti et al. (2011), two major biases are present. First, the authors claim they take the 


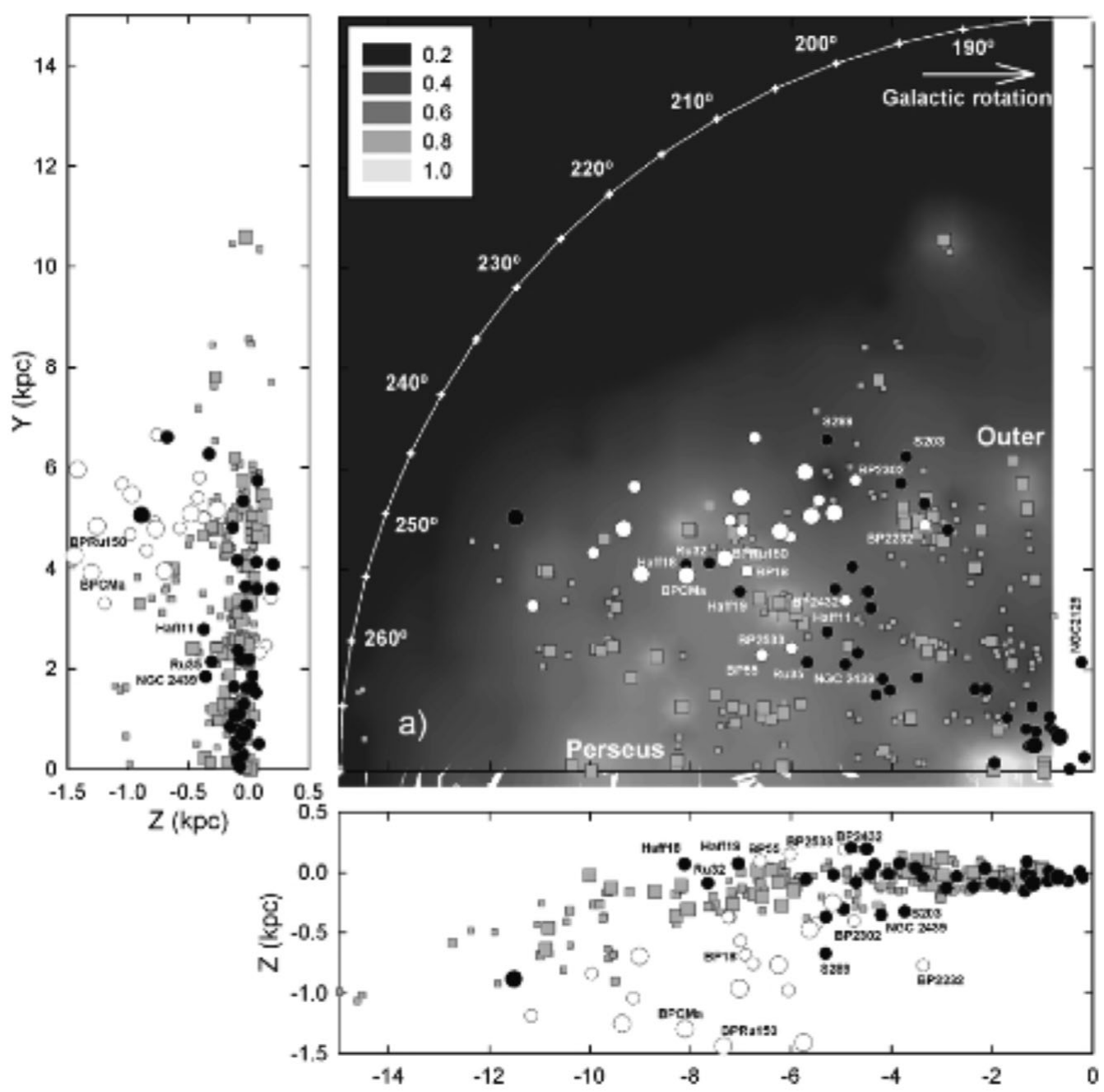

Figure 5. The spiral structure of the Galactic disk in the third quadrant from Vázquez et al. 2008. Solid dots indicate open clusters, while white dots indicate associations of young stars in the background of open star clusters. Finally, squares are for CO clouds. Notice the clear indication of the thin disk warp in the bottom panel.

disk warp into account, still their fields are limited to $-2 \leqslant b \leqslant 2$. The warp is, however, much more extended in latitude (see Momany et al. 2006, and references therein). Second, they use as tracers clump stars. These stars suffer form variable extinction when observed so close to the plane. Momany et al. (2006) exhaustively discussed this point, and clearly showed how Red Giant Branch stars are much better Galactic structure tracers. In fact, in the color magnitude diagram (see Fig. 6), red clump stars form an almost vertical strip, stretched by extinction, that at some point crosses the sub-giant branch stars in the disk. Besides, looking across the disk, a huge amount of variably reddened dwarf stars are also intersected. This implies first that it is very hard to predict the contamination level of the sample, and, second, that a sharp magnitude cut has to be adopted, to avoid confusion. This, in turn, naturally implies a distance cut or limit. Stars more distant than the cut are simply ignored. The detected cut is therefore an artifact of the adopted sample.

To better probe whether the disk has a cut off or not, one should look at the anti-center direction, where extinction is less important. This is what Robin et al. (1992) did, sampling 

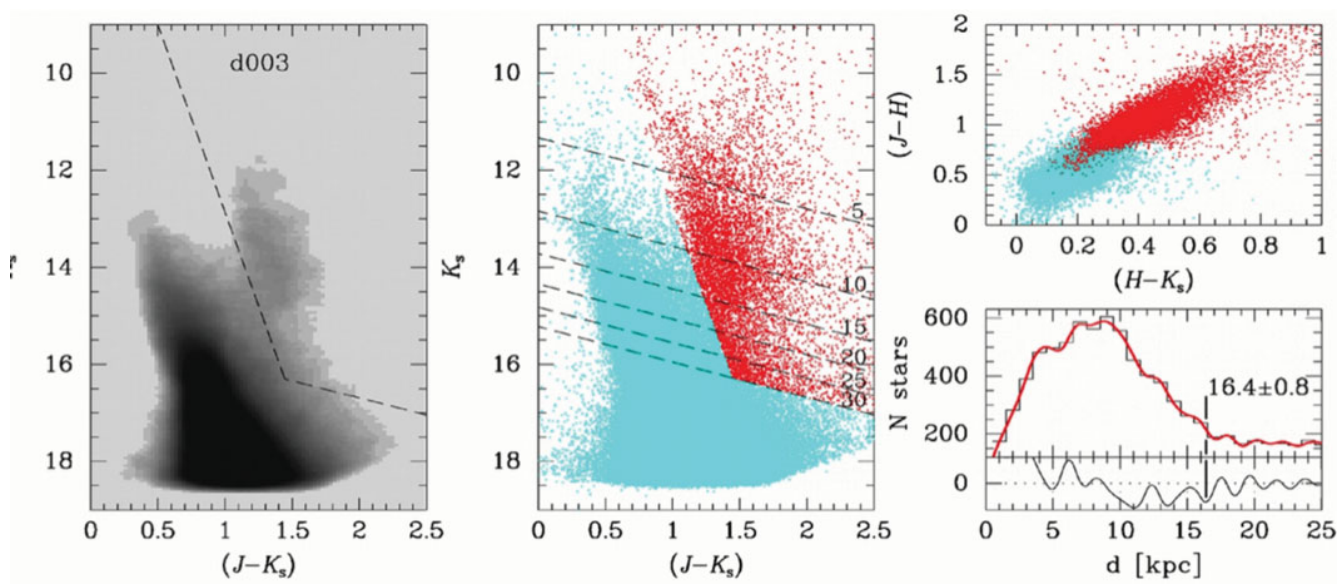

Figure 6. Clump star selection and distance estimates from Minniti et al. (2011). Note the color and magnitude cuts.

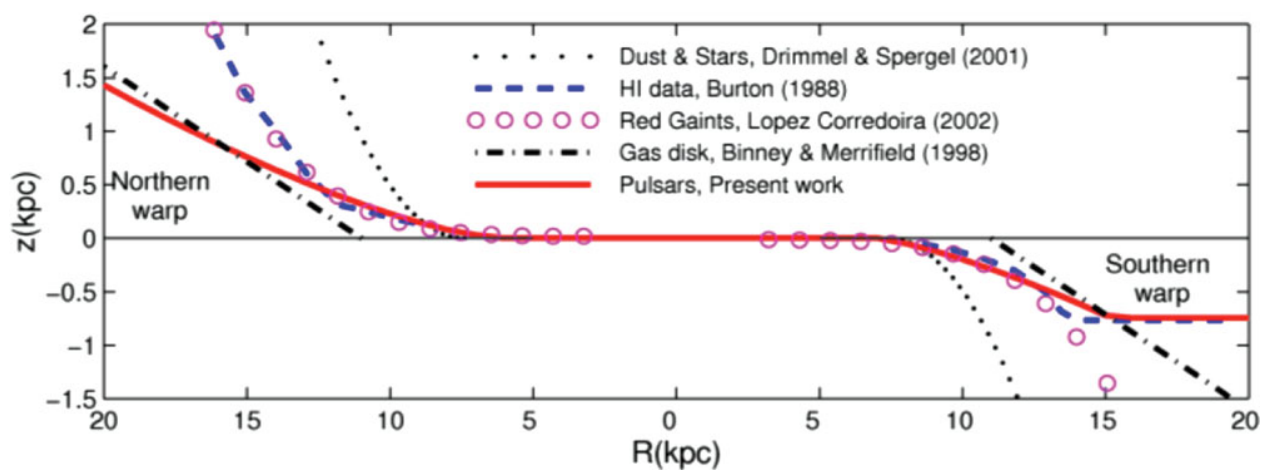

Figure 7. The magnitude and extend of the Galactic warp in the northern and southern disk from various indications from Yusifov (2004). Note how observations limited to low Galactic latitudes ( $\mathrm{Z}=0$ indicates the formal $b=0^{\circ}$ Galactic plane) naturally miss the disk and find erroneously a cut-off.

photometrically a field in the direction of the Galactic anti-center, but close to latitude $b \approx 0$. The cut-off they found is in this case produced by the Galactic warp and flare, as illustrated in Figs. 7 and 8, and it is again artificial. It is natural to find a cut-off when looking along the formal $b=0^{\circ}$ plane, since the disk at a distance of $13-15 \mathrm{kpc}$ from the plane starts bending down. This is quite typical also in many external galaxies.

To follow the disk continuation ones must follow the warp and flare, and therefore the cut-off is simply an illusion caused by the warp and flare. Recent works along this line (Carraro et al. 2010; Sale et al. 2010; Brand \& Wouterloot 2007) indeed find that the stellar disk is much more extended, up to $20 \mathrm{kpc}$ from the Galactic center, and in agreement with HI and HII surveys. In particular, Carraro et al. (2010) found extremely young star associations at more than $20 \mathrm{kpc}$ along the warped disk in the third quadrant.

This implies that the disk is active even at those distances, in agreement with the results of star forming region search in the outer disk of external spirals (Chung et al. 2009; Laine et al. 2013).

A second, quite important conclusion, is that models of Galactic structure need to be revised by removing this artificial cut-off and properly model the Galactic warp and flare. This will help prevent the misinterpretation of observations, like the one of the Canis Major galaxy and the 


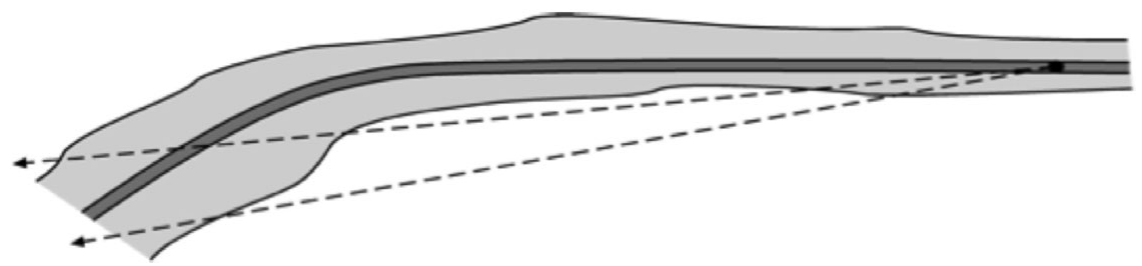

Figure 8. An artistic rendering of the warped Galactic disk as seen from $l=270^{\circ}$. The Galactic center is to the right, the anti-center to the left. The dashed lines indicate the real lines of sight from the Sun (the dot) toward the warped Galactic thin disk (the dark structure) and thick disk (the light structure). This figure is courtesy of Andre Moitinho.

Monoceros ring (Moitinho et al. 2006, Momany et al. 2006, Hammersley \& López-Corredoira 2011, López-Corredoira et al. 2012).

\section{The nature of the Local arm (Orion Spur)}

The Orion spur is the spiral feature inside which our Sun is located. The very inner location of the solar system makes it difficult for us to understand the real nature, orientation, and extent of this structure. It was originally detected by Morgan et al. (1953, see Fig. 1), but its nature has been elusive to present days. It might be a real arm, like Perseus, or a kind of inter-arm feature, like the ones seen in many external spirals. I would like to summarize here recent advances in the field, and propose a new picture of the spiral feature we are immersed in. Recently, $\mathrm{Xu}$ et al. (2012) studied a sample of about 30 masers located in the Orion arm, and measured their trigonometric parallaxes and kinematics. Based on that, they conclude that the kinematic of the sample is typical of a grand design spiral arm, and conclude that the Orion spur is an arm of the same nature of Perseus or Scutum. While the technique is promising, their sources, unfortunately, cover quite a small volume of a few $\mathrm{kpc}$ around the Sun, making it difficult to understand whether the whole Orion spur behave or not like a grand design arm.

A much larger volume has been covered by Moitinho et al. (2006) and Vázquez et al. (2008) using young open clusters and field stars in their background. This study, as already outlined, provides a fresh picture of the spiral structure of the Milky Way in the third quadrant. In particular, it shows that the Local arm penetrates into the third quadrant, and seems to break the Perseus arm before reaching the outer, Norma Cygnus, arm. If this is confirmed, it implies that the Local arm is a much larger structure than currently believed, and behaves like a bridge, connecting the Norma Cygnus arm in the outer disk possibly all the way to the Sagittarius arm in the inner first quadrant of the galaxy. This latter extension has however been poorly explored so far, and much work is warmly recommended. This picture of the Orion spur is not an isolated one: the giant spiral M 74 bears an impressive resemblance to our Milky Way (see fig. 5 in Vázquez et al. 2008).

Intriguingly enough, this picture of the local arm as traced by young open clusters is very similar to the HI realization from Levine et al. (2006). Their fig. 4 shows pretty clearly that a conspicuous Hydrogen structure departs from roughly the Sun location and enters the third quadrant, breaking Perseus and reaching the outer arm.

It is therefore difficult to conceive that the Orion spur is a spiral arm like, e.g., CarinaSagittarius or Scutum-Crux. As a by-product, this result lends support to the idea that the Perseus arm is most probably not a grand design spiral arm.

\section{Conclusions}

In this talk, I reviewed the structure of the thin disk of the Milky Way as traced by young stars and young open clusters. This stellar population traces the disk not only close to the Sun, but all the way to the disk edge in the Galactic anti-center. The expectations from LAMOST and Gaia are very high in this field. For many stars, precise distances will be available and it 
will be possible to position them in the plane of the Galaxy to confirm or deny the actual pieces of evidence for the disk spiral structure and the disk shape and extent.

Specifically, available data on young open cluster and young field stars indicated that the disk is not truncated at $14 \mathrm{kpc}$ from the Galactic center, and that the spiral structure on the outer disk is much different from GLIMPSE expectations. The large-area anti-center coverage of the LAMOST survey will surely provide additional material to improve our understanding on the outer disk.

Besides, the kinematics of millions of stars in the solar vicinity from the Gaia mission will allow one to put firm constraint on the local arm nature and motion, and, hopefully, to understand ultimately its origin.

In the meantime, it is highly recommended to keep accumulating good data on as many young open clusters as possible, for which distances can still be determined precisely. The knowledge of the disk structure is particularly intriguing toward the Galactic center, where - in stars- no clear evidences of spiral structure have been found so far (Perren et al. 2012). Young OB stars seem to show a continuum in their distribution all the way to the Galactic center, with no significant peaks beyond the Carina Sagittarius arm at about $2 \mathrm{kpc}$ from the Sun.

\section{Acknowledgements}

I am mostly indebted to my close collaborators Andre Moitinho and Ruben A. Vázquez. I also benefited from very interesting discussions with Yazan Momany, David Turner, Martin López-Corredoira, and Butler Burton.

\section{References}

Anderson, L. D., Bania, T. M., Balser, D. S., Rood, R. T. 2011, ApJS, 194, 32

Brand, J., \& Wouterloot, J. G.A. 2007, A\&A, 464, 909

Carraro, G., Vázquez, R. A.,Costa, E., Perren, G., Moitinho, A. 2010, ApJ, 718, 683

Carraro, G. 2011, A\&A A, 536, 101

Carraro, G., Beletski, Y., Marconi, G. 2013a, MNRAS, 428, 502

Carraro, G., Turner, D., Majaess, D., Baume, G. 2013b, A\&A, 555, 50

Chung, E. J., Rhee, M.-H., Kin, H., Yun, M. S., Heyer, M. Young, J. S. 2009, ApJS, 184, 199

Churchwell, E., Babler, B. L., Meade, M. R., Whitney, B. A., et al. 2009, PASP, 121, 213

Dame, T. M., Thaddeus, P. 2011, ApJ, 734, L24

de la Fuente Marcos, R., de la Fuente Marcos, C., Moni Bidin, C., Carraro, G., Costa, E. 2013, $M N R A S$, in press

Dias, W. S., Lepine, J. R. D. 2005, ApJ, 629, 825

Gilmore, G., Wyse, R. F.G., Kuijken, K. 1989, ARAA, 27, 555

Gingerich, O. 1985, IAUS, 106, 59

Hammerslery, P. L., López-Corredoira, M. 2011, A\&\&A, 527, A6

Kalberla, P. M.W., \& Kerp, J. 2009, ARAA, 47, 27

Laine, S., Knapen, J. H., Munoz-Mateos, J.-C., Kim, T., et al. 2013, ApJ, in press

Levine, E. S., Blitz, L., Heiles, C. 2006, Science, 312, 1773

Liszt, H. S. 1985, IAUS, 106, 283

López-Corredoira, M., Moitinho, A., Zaggia, S., Momany, Y., Carraro, G., et al. 2012, arXiv, 1207,2749

Majaess, D., Turner, D., Lane, D. J. 2009, JAAVSO,37,179

McClure-Griffiths, N. M., Dickey, J. M., Gaensler, B. M., Green, A. J. 2004, ApJ, 607, L127

Minniti, D., Saito, R. K., Alonso-Garcias, J., Lucas, P. W., Hempel, M. 2011, ApJ, 733, L43

Moitinho, A. 2001, A\&A, 370, 436

Moitinho, A., Vázquez, R. A., Carraro, G.,Baume, G., Giorgi, E. E., Lyra, W. 2006, MNRAS, 368, L77

Momany, Y., Zaggia, S., Gilmore, G., Piotto, G. Carraro, G., Bedin, L., de Angeli, F. 2006, $A \mathscr{E} A, 451,515$ 
Monguió, M., Figueras, F., Grosbol, P. 2012, A\&A, 549, 78

Morgan, W. W., Whitford, A. E., Code, A.,D. 1953, ApJ, 118, 31

Perren, G., Vázquez, R. A., Carraro, G. 2012, A\&A, 548, 125

Reid, M. J., Menten, K. M., Zheng, X. W., Brunthaler, A., et al. 2009, ApJ, 700, 137

Robin, A. C., Creze, M., Mohan, V. 1992, ApJ, 400, L25

Robin, A. C., Reylé,C., Derrieére, S., Picaud, S. 2003, A\&A A, 409, 523

Russeil, D. 2003, A\& A, 397, 133

Sale, S. E., Drew, J. E., Knigge, C., Zijlstra, A. A., et al. 2010, MNRAS, 402, 713

Vázquez, R., May, J., Carraro, G., Bronfman, L., Moitinho, A., Baume, G. 2008, ApJ, 672, 930

Xu, Y., Li, J. J., Reid, M. J., Menten, K. M., et al. 2013, arXiv,1304, 0526

Yusifov, I. 2004, The Magnetized Interstellar Medium, Proceedings of the conference, held in

Antalya, Turkey, September 8 - 12, 2003, Eds: B. Uyaniker, W. Reich, and R. Wielebinski,

Copernicus GmbH, Katlenburg-Lindau., p. 165-169

\section{Discussion}

Teresa Antoja: Some studies suggest that the massive spiral structure traced by old stars induces two additional spiral arms made of gas and stars. Do you think we have enough evidence for that? What do you think of that idea?

Giovanni Carraro: I believe spiral arms regenerate continuously. In the case of the Milky Way we do not see differences in young star and star clusters among the so-called massive arms (Perseus and Scutum) and the so-called secondary arms (Carina and Norma). The difference is apparently seen only in HI, although different surveys produce different results.

JAMEs Binney: I think that N-body simulations make it clear that spiral structure is ephemeral. But it does not from this follow that it cannot be traced by old object because spiral structure is written in the gravitational potential. This marshall gas, causing it to shock and form stars, but also marshall stars of all types, causing them to linger along arms. So yes very young objects are good tracers of spiral arms via star formation, but older stars that have smallish velocity dispersion can also be good tracers.

Giovanni Carraro: I think the argument of the gravitational trapping is mostly correct. However to conclude that old stars trace spiral arms is incorrect and dangerous in my point of view. Old stars are mostly spread everywhere, while young stars and gas are preferentially located inside spiral arms, and therefore are the best spiral arm tracers. In the specific case of star counts (GLIMPSE), there is no way to distinguish small velocity dispersion from high velocity dispersion clump stars, one sums up everything, and therefore the outcome is not completely reliable. I would tend to believe more in the distribution of the younger material. 